\title{
New developments on structure formation in Chaplygin gas cosmological models
}

\author{
Sandro Silva e Costa* \\ $C C N H$, Universidade Federal do ABC, Brazil \\ E-mail: sandro.costa@ufabc.edu.br

\section{Marceliano Eduardo de Oliveira} \\ Instituto de Física, ICET, Universidade Federal de Mato Grosso, Brazil \\ E-mail: marceliano@cpd.ufmt.br
}

\begin{abstract}
In this work we show that the use of a cosmological model with a non-linear equation of state can produce a universe with expansion, and in such context we examine the structure formation in Chaplygin gas cosmological models. Our focus is on the new modified Chaplygin gas model, which can present exact analytical solutions for the density contrast and for the power spectrum for some particular values of its parameters.
\end{abstract}

5th International School on Field Theory and Gravitation April 20-24, 2009

Cuiabá city, Brazil

${ }^{*}$ Poster Session 


\section{Introduction}

Distinct cosmological models can lead to a 'degenerate' behaviour of the scale factor $a(t)$, i.e., different ideas such as viscosity and a dynamical cosmological constant can yield the same kind of solution for $a(t)$. Therefore, a possible way of distinhguish between these models would be to observe which influence they have in the evolution of density perturbations in the primordial universe. The basic idea is that the universe began almost perfectly homogeneous, with small fluctuations in the density of matter in some places. By influence of gravity and of the expansion of universe such fluctuations or perturbations of the homogeinity can be wiped out - decreasing modes - or increased in size - increasing modes. The increasing modes are the ones who gave birth to the non-homogenous structures observed today (galaxies, clusters and so on) [1].

The behaviour of the density fluctuations depends on $a(t)$. However, a relativistic equation must also include information on some thermodynamical quantities, such as the pressure of the cosmological fluid in use in the model, $p$, or the density of energy of the fluid, $\rho$, and relations between such quantities. In the case where there is no curvature (plane space) the differential equation for the Fourier mode of wavemode $k$ of a density perturbation $\delta$ is [2]

$$
\frac{d^{2} \delta}{d a^{2}}+\frac{3}{2 a}\left(1-5 w+2 v^{2}\right) \frac{d \delta}{d a}-\frac{3 \delta}{2 a^{2}}\left(1-6 v^{2}-3 w^{2}+8 w\right)=-\frac{k^{2} v^{2}}{H^{2} a^{4}} \delta .
$$

where $H=\dot{a} / a, w=p / \rho$ and $v^{2}=\frac{\partial p}{\partial \rho}$, i.e., one has a dependency on the equation of state $p=p(\rho)$ of the cosmological fluid in study.

The simplest models are obtained considering only fluids with a linear equation of state,

$$
p=(\gamma-1) \rho,
$$

where $\gamma$ is a parameter of the fluid. However, one can also consider fluids with a non-linear term, where

$$
p=(\gamma-1) \rho+f(\rho)
$$

\section{Models with a single exotic non-linear fluid}

Any cosmological model with a single fluid must obey the equation for energy conservation

$$
\dot{\rho}+3 H(\rho+p)=0,
$$

and the Friedmann equation

$$
H^{2}+\frac{k}{a^{2}}=\frac{8 \pi}{3} \rho .
$$

After some algebra one can write this as [3]

$$
\dot{H}+\frac{3 \gamma}{2} H^{2}+\frac{k}{a^{2}}\left(\frac{3 \gamma}{2}-1\right)=-4 \pi f(\rho),
$$

or, if $\gamma \neq 0$

$$
\frac{d^{2}}{d t^{2}}\left(a^{\frac{3 \gamma}{2}}\right)+k \frac{3 \gamma}{2}\left(\frac{3 \gamma}{2}-1\right) a^{\frac{3 \gamma}{2}-2}=-6 \pi \gamma a^{\frac{3 \gamma}{2}} f(\rho)
$$


while for $\gamma=0$ one has

$$
\frac{d^{2}}{d t^{2}}(\ln a)-\frac{k}{a^{2}}=-4 \pi f(\rho)
$$

If, for example, one has, for $\gamma \neq 0$,

$$
f(\rho)=\alpha H+\beta
$$

or, for $\gamma=0$,

$$
f(\rho)=\alpha H+\beta \ln a,
$$

where $\alpha$ and $\beta$ are constants, one has, for $\gamma \neq 0$,

$$
\frac{d^{2}}{d t^{2}}\left(a^{\frac{3 \gamma}{2}}\right)+4 \pi \alpha \frac{d}{d t}\left(a^{\frac{3 \gamma}{2}}\right)+6 \pi \beta \gamma a^{\frac{3 \gamma}{2}}=-k \frac{3 \gamma}{2}\left(\frac{3 \gamma}{2}-1\right) a^{\frac{3 \gamma}{2}-2}
$$

or, when $\gamma=0$,

$$
\frac{d^{2}}{d t^{2}}(\ln a)+4 \pi \alpha \frac{d}{d t}(\ln a)+4 \pi \beta(\ln a)=\frac{k}{a^{2}} .
$$

From these two last equations one can see that if $k=0$ (plane space) and $\alpha<0$ these equations are similar to the ones for an harmonic oscillator 'damped' by an anti-friction term. Therefore the non-linear term $f(\rho)$ can yield solutions for the scale factor that represent an expanding universe.

\section{The new modified Chaplygin gas model}

The new modified Chaplygin gas model (NMCG) is a specific case where one has [4]

$$
f(\rho)=f(\rho, a)=-M a^{-v} \rho^{-\mu},
$$

i.e.,

$$
p=(\gamma-1) \rho-M a^{-v} \rho^{-\mu},
$$

where $\gamma, M, v$ and $\mu$ are free parameters of the model. With this, one can also have

$$
\rho=\left[A a^{-v}+(B-A) a^{-3 \mu(1+\mu)}\right]^{\frac{1}{1+\mu}}
$$

where $A$ and $B$ are constants.

In such case the equation for the perturbations is

$$
\begin{aligned}
& \frac{d^{2} \delta}{d z^{2}}-\frac{1}{z}\left[\frac{\frac{10}{3}-3 \gamma}{\gamma-\gamma_{x}} x-1+\frac{3 x+1}{1-z}+\frac{\frac{\gamma_{x}}{\gamma}}{z-\frac{\gamma_{x}}{\gamma}}\right] \frac{d \delta}{d z}+ \\
& \frac{k^{2} x z^{\frac{2 x}{3}\left(\frac{2-3 \gamma_{x}}{\gamma-\gamma_{x}}\right)-2}}{6 \pi A^{2 x} y^{2-3 \gamma_{x}}\left(\gamma-\gamma_{x}\right)(1-z)^{2 x}}\left[\frac{\gamma-1}{\gamma-\gamma_{x}} x+\frac{\frac{1}{2}-x}{1-z}+\frac{\frac{\gamma_{x}}{2 \gamma}}{z-\frac{\gamma_{x}}{\gamma}}\right] \delta= \\
& \frac{2 x \delta}{\left(\gamma-\gamma_{x}\right) z^{2}}\left[\frac{(2-\gamma)\left(\gamma-\frac{2}{3}\right)}{\left(\gamma-\gamma_{x}\right)} x-\frac{\frac{8 x}{3}+1-2 x \gamma}{1-z}-\frac{\gamma-\gamma_{x}}{(1-z)^{2}} x-\frac{\frac{\gamma_{x}}{\gamma}}{z-\frac{\gamma_{x}}{\gamma}}\right] .
\end{aligned}
$$

where $z=-\left(\frac{B-A}{A}\right) a^{v-3 \gamma(1+\mu)}, y=\left[-\frac{B-A}{A}\right]^{\frac{2 x}{3\left(\gamma-\gamma_{x}\right)}} 2 x=(1+\mu)^{-1}$ and $\gamma_{x}=2 x v / 3$. 


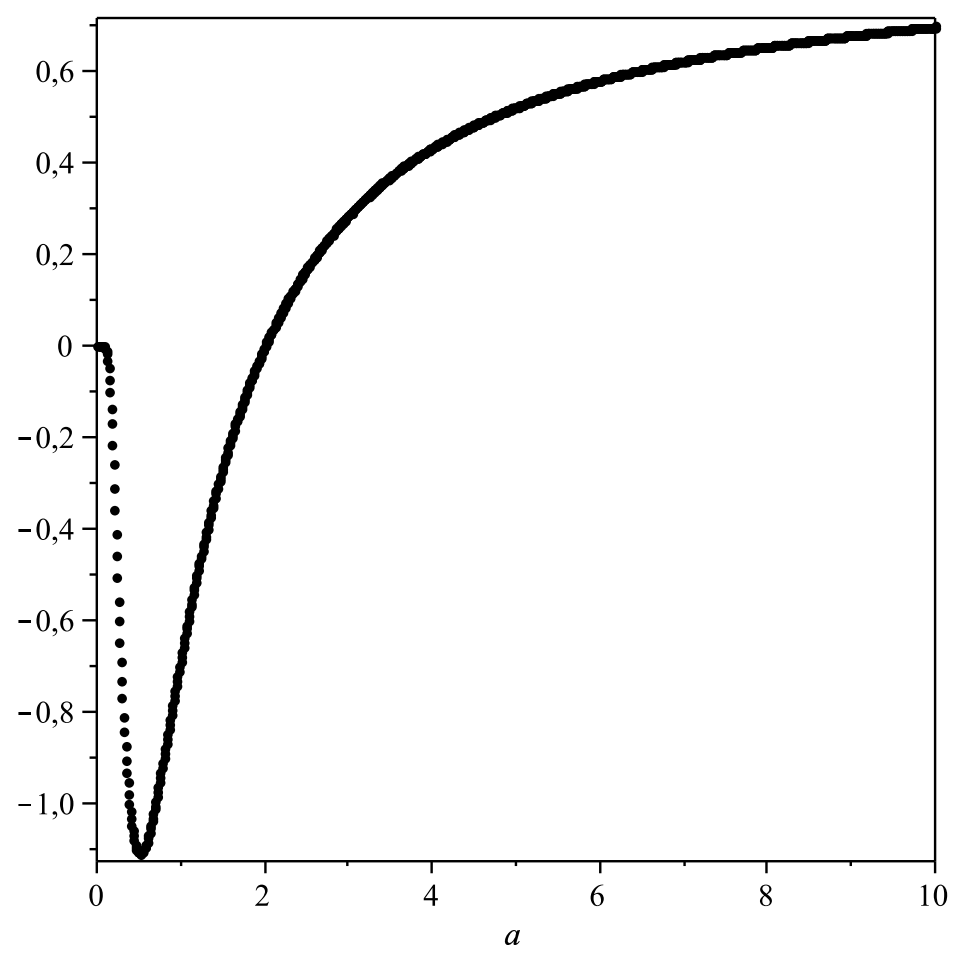

Figure 1: Graphic representation of a solution well-behaved at the origin for the density contrast $\delta$ as a function of the scale factor $a$, for the NMCG with $\gamma=0, \gamma_{x}=2 / 3, x=-1$ and an arbitrary value of $\tilde{k}$.

For some particular cases such equation has exact analytical solutions. One example of this situation is when $\gamma=0$ and $\gamma_{x}=2 / 3$, when a solution is obtained in terms of Whittaker functions [5],

$$
M_{\lambda, \mu}(u)=u^{\mu+\frac{1}{2}} e^{-\frac{u}{2}} \Phi\left(\mu-\lambda+\frac{1}{2}, 2 \mu-1 ; u\right),
$$

with $\Phi(\alpha, \gamma ; z)$ being the confluent hypergeometric function. Then, one has

$$
\delta(a)=\left(\frac{1}{a^{\frac{1}{2}}}-\frac{\beta}{a^{\frac{3}{2}}}\right)\left[c_{1} M_{-\frac{5 \tilde{k}}{16}, \sqrt{1-\frac{\tilde{k}^{2}}{16}}}\left(-\frac{\tilde{k} \beta}{a}\right)+c_{2} M_{-\frac{5 \tilde{k}}{16},-\sqrt{1-\frac{\tilde{k}^{2}}{16}}}\left(-\frac{\tilde{k} \beta}{a}\right)\right]
$$

where now $\beta \equiv 1+\frac{2}{3 M \rho_{0}^{1 / 2}}$ and $\tilde{k} \equiv \frac{3 M k}{\sqrt{2 \pi}}$. Such solutions can be represented graphically, and from them one can easily obtain the power spectrum $P=P(k)$, since $P \propto|\delta|^{2}$. An example of the final result obtained for the density contrast as a function of the scale factor is presented in Figure 1, and the power spectrum obtained from it is presented in Figure 2.

\section{Conclusion}

In this work we have shown that the use of a cosmological model with a non-linear equation of state can produce a universe with expansion, i.e., where the scale factor grows as accelerated by an anti-friction term. One of these models is the so-called new modified Chaplygin gas (NMCG), which has four freee parameters, and as such gives a plethora of possibilities. When density perturbations are studied in the context of the NMCG, one can obtain, in some cases, analytical solutions 


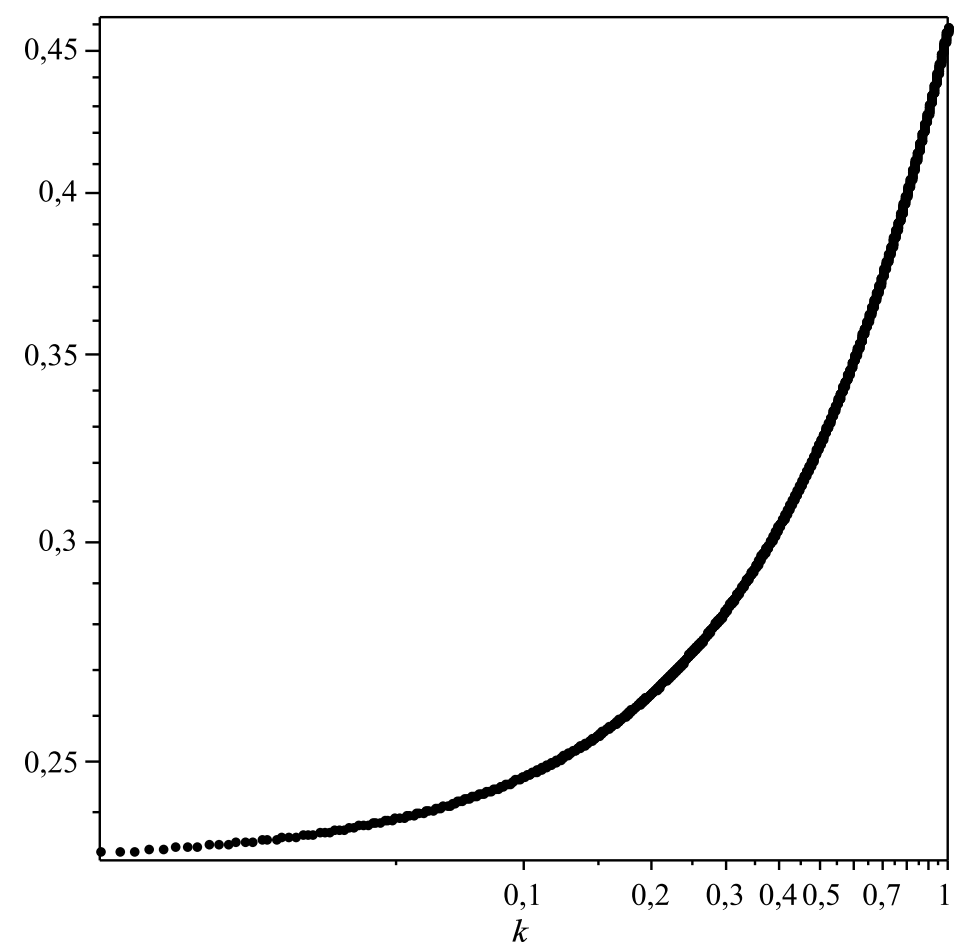

Figure 2: Graphic representation of the power spectrum $P=P(k)$ obtained from the solution for the density contrast $\delta$ for the NMCG with $\gamma=0, \gamma_{x}=2 / 3$ and $x=-1$.

from where one can easily obtain the power spectrum associated. Such results indicate that more complete studies on the possibilities open by the NMCG must be done in order to observe, in some cases, its possible adequacy to explain the observed features of the universe.

\section{References}

[1] B. Ryden, Introduction to cosmology, Addison-Wesley, San Francisco 2003.

[2] T. Padmanabhan, Structure formation in the universe, Cambridge University Press, Cambridge 1993.

[3] S. S. e Costa and M. Makler, Connections among three roads to cosmic acceleration: decaying vacuum, bulk viscosity, and nonlinear fluids, arXiv: astro-ph/0702418v1.

[4] S. Chattopadhyay and U. Debnath, Density evolution in the new modified Chaplygin gas model, Grav. and Cosmol. 14 (2008) 341 [arXiv: 0805.0070v1 [gr-qc] ].

[5] I. S. Gradshteyn and I. M. Ryzhik, Table of integral, series and products, 6th. ed., Academic Press, New York 2000. 